\title{
Renormalization group in difference systems
}

\author{
M Iwasa and K Nozaki \\ Department of Physics, Nagoya university, Nagoya 464-8602, Japan \\ E-mail: miwasa@r.phys.nagoya-u.ac.jp
}

\begin{abstract}
.
A new singular perturbation method based on the Lie symmetry group is presented to a system of difference equations. This method yields consistent derivation of a renormalization group equation which gives an asymptotic solution of the difference equation. The renormalization group equation is a Lie differential equation of a Lie group which leaves the system approximately invariant. For a 2-D symplectic map, the renormalization group equation becomes a Hamiltonian system and a long-time behaviour of the symplectic map is described by the Hamiltonian. We study the Poincaré-Birkoff bifurcation in the 2-D symplectic map by means of the Hamiltonian and give a condition for the bifurcation.
\end{abstract}

PACS numbers: $02.20-\mathrm{a}, 02.30 \mathrm{Mv}, 02.30 \mathrm{Oz}, 02.40 \mathrm{Xx}, 45.10 \mathrm{Hj}$

\section{Introduction}

There have been many studies concerning application of renormalization group method of quantum field theory as a singular perturbation method to treat differential equations since the work of Illinois groups [1] 2]. Although this method enables us to remove secular or divergent terms appearing in a naive perturbation solution by renormalizing integral constants appearing in the lowest-order of perturbation solution and to give a well-behaved asymptotic solution, it is necessary to calculate a naive perturbation solution to derive the renormalization group equation, and whether the system could be renormalized or not depends on the functional form of a naive perturbation solution. The present authors presented another renormalization group method in terms of the Lie symmetry group [3. Without calculating a naive perturbation solution, the renormalized solution is constructed as an invariant solution under transformation which leaves the system approximately invariant. The approximate Lie group transforms an unperturbed solution into a renormalized perturbed solution. The both renormalization group methods were based on the theory of continuous group and so they were developed to apply a system of differential equations. For some discrete dynamical systems such as a system of difference equations, there has been found a working procedure to derive a renormalized difference equation by means of a naive perturbation solution [4] [5]. However, the concept of group was not used in the procedure to derive a renormalized 
discrete system and so legitimacy of the result is not clear. The purpose of this paper is to develop a renormalization group method in terms of the Lie symmetry group for a system of difference equations. Apparently, it seems to be difficult to use the same procedure as in the case of a differential equation when we treat a discrete system because Lie symmetry groups are one-parameter continuous groups. However, we regard a discrete system as an algebraic system and consider a particular class of approximate Lie symmetry groups which transforms a perturbed system to an unperturbed system approximately. Then we succeed in obtaining a renormalized perturbed solution by means of the so-called Lie differential equation corresponding to the approximate Lie symmetry. Thus, we present a renormalization group method for a system of difference equations, where the continuous group theory is fully employed, and the Lie equation becomes the renormalization group equation for a difference system. As an application of the new method, we study the Poincaré-Birkoff bifurcation in a 2-D symplectic map and give a condition for the bifurcation explicitly.

\section{Renormalization group method with Lie symmetry}

Let us consider the following 2-D symplectic map of action-angle type, $\left(u_{n}, v_{n}\right) \mapsto$ $\left(u_{n+1}, v_{n+1}\right)$ :

$$
\begin{aligned}
& u_{n+1}=u_{n}+v_{n+1}, \\
& v_{n+1}=v_{n}+a u_{n}+\varepsilon g\left(u_{n}\right),
\end{aligned}
$$

where the coefficient $a \in \mathbb{R}$ is constant, $g$ is a function of $u_{n}$, and $\varepsilon$ is a perturbation parameter which is small. The system (1) reads

$$
\left(\begin{array}{l}
u_{n+1} \\
v_{n+1}
\end{array}\right)=\left(\begin{array}{cc}
a+1 & 1 \\
a & 1
\end{array}\right)\left(\begin{array}{l}
u_{n} \\
v_{n}
\end{array}\right)+\left(\begin{array}{l}
\varepsilon g\left(u_{n}\right) \\
\varepsilon g\left(u_{n}\right)
\end{array}\right) .
$$

Under a transformation of coordinates $\left(u_{n}, v_{n}\right) \mapsto\left(z_{n}, \overline{z_{n}}\right)$ such as

$$
\begin{aligned}
& \left(\begin{array}{c}
z_{n} \\
z_{n}
\end{array}\right)=\left(\begin{array}{cc}
\frac{1}{2 \cos (\omega / 2)} & \mathrm{i} \frac{\exp (\mathrm{i} \omega / 2)}{2 \sin \omega \cos \omega} \\
\frac{1}{2 \cos (\omega / 2)} & -\mathrm{i} \frac{\exp (-\mathrm{i} \omega / 2)}{2 \sin \omega \cos \omega}
\end{array}\right)\left(\begin{array}{c}
u_{n} \\
v_{n}
\end{array}\right), \\
& \Longleftrightarrow\left(\begin{array}{c}
u_{n} \\
v_{n}
\end{array}\right)=\left(\begin{array}{cc}
\exp (-\mathrm{i} \omega / 2) & \exp (\mathrm{i} \omega / 2) \\
-2 \mathrm{i} \sin (\omega / 2) & 2 \mathrm{i} \sin (\omega / 2)
\end{array}\right)\left(\begin{array}{c}
z_{n} \\
\overline{z_{n}}
\end{array}\right),
\end{aligned}
$$

the linear part of (2) is diagonalized as follows:

$$
\left(\begin{array}{c}
z_{n+1} \\
z_{n+1}
\end{array}\right)=\left(\begin{array}{cc}
\mathrm{e}^{-\mathrm{i} \omega} & 0 \\
0 & \mathrm{e}^{\mathrm{i} \omega}
\end{array}\right)\left(\begin{array}{c}
z_{n} \\
z_{n}
\end{array}\right)+\varepsilon \frac{g\left(u_{n}\right)}{2 \sin \omega}\left(\begin{array}{c}
\mathrm{i} \exp (-i \omega / 2) \\
-\mathrm{i} \exp (i \omega / 2)
\end{array}\right),
$$

where $a+2=: 2 \cos \omega$, and $\overline{z_{n}}$ denotes the complex conjugate of $z_{n}$. Here $0<a<-4$ because we are interested in a case the origin is assumed to be elliptic.

Since the second component of (5) is the complex conjugate of the first, we have only to consider the first component of the equation, which is

$$
z_{n+1}=\mathrm{e}^{-\mathrm{i} \omega} z_{n}+\varepsilon \frac{\mathrm{i} \exp (-\mathrm{i} \omega / 2)}{2 \sin \omega} g\left(u_{n}\right) .
$$


Let (6) admit a Lie group transformation whose infinitesimal generator takes the form

$$
\begin{aligned}
X\left(n, z_{n}, \overline{z_{n}}, z_{n+1}, \overline{z_{n+1}}\right)=\partial_{\varepsilon}+\eta^{z}\left(n, z_{n}, \overline{z_{n}}\right) \partial_{z_{n}}+\eta^{\bar{z}}\left(n, z_{n}, \overline{z_{n}}\right) \partial_{\overline{z_{n}}} \\
+\eta^{z}\left(n+1, z_{n+1}, \overline{z_{n+1}}\right) \partial_{z_{n+1}}+\eta^{\bar{z}}\left(n+1, z_{n+1}, \overline{z_{n+1}}\right) \partial_{\overline{z_{n+1}}}
\end{aligned}
$$

We can rewrite (7) to

$$
\begin{aligned}
& X\left(n, z_{n}, \overline{z_{n}}, z_{n+1}, \overline{z_{n+1}}\right)=\partial_{\varepsilon}+\eta\left(n, z_{n}, \overline{z_{n}}\right) \partial_{z_{n}}+\overline{\eta\left(n, z_{n}, \overline{z_{n}}\right)} \partial_{\overline{z_{n}}} \\
& +\eta\left(n+1, z_{n+1}, \overline{z_{n+1}}\right) \partial_{z_{n+1}}+\overline{\eta\left(n+1, z_{n+1}, \overline{z_{n+1}}\right)} \partial_{\overline{z_{n+1}}}
\end{aligned}
$$

because it can be shown that $\eta^{\bar{z}}=\overline{\eta^{z}}$. Then the determining equation for (6), which determines $\eta\left(n, z_{n}, \overline{z_{n}}\right)$ of the vector field (요 [6] , is given by

$\left.X\left(n, z_{n}, \overline{z_{n}}, z_{n+1}, \overline{z_{n+1}}\right)\left\{z_{n+1}-\mathrm{e}^{-\mathrm{i} \omega} z_{n}-\varepsilon \frac{\mathrm{i} \exp (-\mathrm{i} \omega / 2)}{2 \sin \omega} g\left(u_{n}\right)\right\}\right|_{\text {Equation (6) }}=0$.

Because we wish to find such a symmetry that leaves the system approximately invariant to leading order, we need only to solve the following leading-order determining equation:

$$
\begin{array}{r}
\left.X\left(n, z_{n}, \overline{z_{n}}, z_{n+1}, \overline{z_{n+1}}\right)\left\{z_{n+1}-\mathrm{e}^{-\mathrm{i} \omega} z_{n}-\varepsilon \frac{\mathrm{i} \exp (-\mathrm{i} \omega / 2)}{2 \sin \omega} g\left(u_{n}\right)\right\}\right|_{z_{n+1}=\mathrm{e}^{-\mathrm{i} \omega} z_{n}}=\mathrm{O}(\varepsilon), \\
\Longleftrightarrow \eta\left(n+1, \mathrm{e}^{-\mathrm{i} \omega} z_{n}, \mathrm{e}^{\mathrm{i} \omega} \overline{z_{n}}\right)-\mathrm{e}^{-\mathrm{i} \omega} \eta\left(n, z_{n}, \overline{z_{n}}\right)=\frac{\mathrm{i} \exp (-\mathrm{i} \omega / 2)}{2 \sin \omega} g\left(u_{n}\right) .
\end{array}
$$

By solving (11), we obtain the infinitesimal generator $X$ admitted by the system (6) in the leading-order approximation.

Using the obtained infinitesimal generator $X$, we construct a group-invariant solution of the system (6) $)$. The group-invariant solution, $z_{n}=z_{n}(\varepsilon)$, satisfies the following Lie equation:

$$
\left.X\left\{z_{n}-z_{n}(\varepsilon)\right\}\right|_{z_{n}=z_{n}(\varepsilon)}=0,
$$

which reads

$$
\frac{\mathrm{d} z_{n}(\varepsilon)}{\mathrm{d} \varepsilon}=\eta\left(n, z_{n}(\varepsilon), \overline{z_{n}(\varepsilon)}\right) .
$$

We refer to the Lie equation (13) as the renormalization group equation. Solving the renormalization group equation by adopting solutions of unperturbed system as a boundary condition, i.e.

$$
z_{n}(\varepsilon=0)=z_{n}^{(0)}
$$

where $z_{n}=z_{n}^{(0)}$ denotes the solution of the unperturbed system, we obtain an asymptotic solution of the system.

\section{Power series nonlinear terms}

Let us consider a case $g\left(u_{n}\right)$ is a power series with respect to $u_{n}$, i.e. we set

$$
g\left(u_{n}\right):=\sum_{j=0}^{\infty} A_{j} u_{n}^{j}
$$


where $A_{j} \in \mathbb{R}$. Under the linear transformation (44), (15) can be written as

$$
g\left(u_{n}\left(z_{n}, \overline{z_{n}}\right)\right)=\sum_{l, m} B_{l m}(\omega) z_{n}^{l}{\overline{z_{n}}}^{m}
$$

where

$$
B_{l m}(\omega)=A_{l+m} l+m C_{l} \exp [-\mathrm{i}(l-m) \omega / 2] .
$$

Here ${ }_{k} C_{r}:=k ! /[r !(k-r) !]$. Then the determining equation (11) becomes

$\eta\left(n+1, e^{-\mathrm{i} \omega} z_{n}, e^{\mathrm{i} \omega} \overline{z_{n}}\right)-e^{-\mathrm{i} \omega} \eta\left(n, z_{n}, \overline{z_{n}}\right)=\frac{\mathrm{i} \exp (-\mathrm{i} \omega / 2)}{2 \sin \omega} \sum_{l, m} B_{l m}(\omega) z_{n}^{l}{\overline{z_{n}}}^{m}$.

The nature of the solution of (18) depends on whether $\omega / 2 \pi$ is rational or irrational.

\section{1. $\omega / 2 \pi$ is irrational}

Let $\omega / 2 \pi$ be irrational, then only terms proportional to $z_{n}^{l+1} \overline{z_{n}} l$ cause resonance, and the solution of the determining equation (18) becomes

$$
\begin{aligned}
\eta\left(n, z_{n}, \bar{z}_{n}\right)= & \sum_{l, m \text { s.t. } l-m=1} \frac{\mathrm{i} \exp (\mathrm{i} \omega / 2) B_{l m}(\omega)}{2 \sin \omega} n z_{n}^{l}{\overline{z_{n}}}^{m} \\
& +\sum_{l, m \text { s.t. } l-m \neq 1} \frac{\mathrm{i} \exp (-\mathrm{i} \omega / 2) B_{l m}(\omega)}{2 \sin \omega\{\exp [-\mathrm{i}(l-m) \omega]-\exp [-\mathrm{i} \omega]\}} z_{n}^{l}{\overline{z_{n}}}^{m} .
\end{aligned}
$$

Here $\exp [-\mathrm{i}(l-m) \omega]-\exp [-\mathrm{i} \omega] \neq 0$ for $l-m \neq 1$. The terms proportional to $n$ are called secular terms. The renormalization group equation i.e. (13) becomes

$$
\begin{aligned}
\frac{\mathrm{d} z_{n}}{\mathrm{~d} \varepsilon}= & \sum_{l, m \text { s.t. } l-m=1} \frac{\mathrm{i} \exp (\mathrm{i} \omega / 2) B_{l m}(\omega)}{2 \sin \omega} n z_{n}^{l}{\overline{z_{n}}}^{m} \\
& +\sum_{l, m \text { s.t. } l-m \neq 1} \frac{\mathrm{i} \exp (\mathrm{i} \omega / 2) B_{l m}(\omega)}{2 \sin \omega\{\exp [-\mathrm{i}(l-m) \omega]-\exp [-\mathrm{i} \omega]\}} z_{n}^{l}{\overline{z_{n}}}^{m} .
\end{aligned}
$$

Because we are interested in a long-time behaviour of the system, we consider the case of $n \gg 1$. Then we neglect non-secular terms and the renormalization group equation becomes

$$
\begin{aligned}
\frac{\mathrm{d} z_{n}}{\mathrm{~d} \varepsilon} & =\frac{\mathrm{i} \exp (\mathrm{i} \omega / 2)}{2 \sin \omega} \sum_{l=0}^{\infty} B_{l+1}(\omega) n\left|z_{n}\right|^{2 l} z_{n}, \\
& =\frac{\mathrm{i}}{2 \sin \omega} \sum_{l=0}^{\infty} A_{2 l+1}{ }_{2 l+1} C_{l} n\left|z_{n}\right|^{2 l} z_{n},
\end{aligned}
$$

where we use (17). Because $\mathrm{d}\left|z_{n}\right| / \mathrm{d} \varepsilon=0$, introducing $R:=\left|z_{n}\right|$, the solution of (21) becomes

$$
z_{n}(\varepsilon)=z_{n}(0) \exp \left(\frac{\mathrm{i}}{2 \sin \omega} \sum_{l=0}^{\infty} A_{2 l+1} 2 l+1 C_{l} R^{2 l} n \varepsilon\right) .
$$

Thus, we obtain an asymptotic behaviour of a difference equation by solving a differential equation. 


\section{2. $\omega / 2 \pi$ is rational}

Let $\omega / 2 \pi$ be rational i.e. $\omega=2 \pi q / p$ where $q, p \in \mathbb{N}$, then denominators of the second terms in the right hand side of (20), $\exp [-\mathrm{i}(l-m) \omega]-\exp [-\mathrm{i} \omega]$, become zero when $l-m=1+k p$ for $k \in \mathbb{Z}$. Therefore terms $z_{n}^{l} \bar{z}_{n}{ }^{m}$ where $l-m=1+k p$ give secular contribution to the determining equation (18). The solution of the determining equation becomes

$$
\begin{aligned}
\eta\left(n, z_{n}, \bar{z}_{n}\right)= & \sum_{l, m \text { s.t. } l-m=1+k p} \frac{\mathrm{i} \exp (\mathrm{i} \omega / 2) B_{l m}(\omega)}{2 \sin \omega} n z_{n}^{l} \bar{z}_{n}^{m} \\
& +\sum_{l, m \text { s.t.l }-m \neq 1+k p} \frac{\mathrm{i} \exp (-\mathrm{i} \omega / 2) B_{l m}(\omega)}{2 \sin \omega\{\exp [-\mathrm{i}(l-m) \omega]-\exp [-\mathrm{i} \omega]\}} z_{n}^{l}{\overline{z_{n}}}^{m} .
\end{aligned}
$$

In the case of $n \gg 1$, the renormalization group equation reads

$$
\frac{\mathrm{d} z_{n}}{\mathrm{~d} \varepsilon}=\sum_{l, m \text { s.t. } l-m=1+k p} \frac{\mathrm{i} \exp (\mathrm{i} \omega / 2) B_{l m}(\omega)}{2 \sin \omega} n z_{n}^{l}{\overline{z_{n}}}^{m},
$$

which is rewritten as

$$
\begin{aligned}
\frac{\mathrm{d} z_{n}}{\mathrm{~d} \varepsilon}=\frac{\mathrm{i} \exp (\mathrm{i} \omega / 2) n}{2 \sin \omega}[ & \sum_{l \geq 1, m \geq 0} B_{l+m p} l-1(\omega)\left|z_{n}\right|^{2(l-1)} z_{n}^{m p+1} \\
& \left.+\sum_{l \geq 0, m \geq 1} B_{l}{ }_{l+m p-1}(\omega)\left|z_{n}\right|^{2 l}{\overline{z_{n}}}^{m p-1}\right],
\end{aligned}
$$

or

$$
\begin{aligned}
& \frac{\mathrm{d} z_{n}}{\mathrm{~d} \tau}= \frac{\mathrm{i} \exp (\mathrm{i} \omega / 2)}{2 \sin \omega}\left[\sum_{l \geq 1, m \geq 0} B_{l+m p} l-1(\omega)\left|z_{n}\right|^{2(l-1)} z_{n}^{m p+1}+\sum_{l \geq 0, m \geq 1} B_{l} l+m p-1(\omega)\left|z_{n}\right|^{2 l} \bar{z}_{n}{ }^{m p-1}\right], \\
&= \frac{\mathrm{i}}{2 \sin \omega}\left[\sum_{l \geq 1, m \geq 0} A_{2 l+m p-1} 2 l+m p-1 C_{l+m p}\left|z_{n}\right|^{2(l-1)} z_{n}^{m p+1}\right. \\
&+\sum_{l \geq 0, m \geq 1} A_{2 l+m p-1} 2 l+m p-1 \\
&\left.C_{l}\left|z_{n}\right|^{2 l} \bar{z}_{n} m p-1\right],
\end{aligned}
$$

where $\tau:=\varepsilon n$. This is a Hamiltonian system whose Hamiltonian is

$$
H\left(z_{n}, \overline{z_{n}}\right)=\sum_{\substack{l \geq 0, m \geq 0 \\(l, m) \neq(0,0)}}\left[\mathrm{i} A_{2 l+m p-1} \frac{(2 l+m p-1) !}{(l+m p) ! l !}\left|z_{n}\right|^{2 l}\left(z_{n}^{m p}+{\overline{z_{n}}}^{m p}\right)\right] .
$$

Because (26) describes also $n$ dependence of $z_{n}(\varepsilon)$, the phase space structure of this Hamiltonian system provides a long-time behaviour of the difference equation.

\section{3. $\omega / 2 \pi$ is close to a rational number}

In order to study the Poincaré-Birkoff bifurcation in the symplectic map, let us consider the case $\omega / 2 \pi$ is close to a rational number,

$$
\omega=\omega_{0}+\varepsilon \delta,
$$


where $\omega_{0} / 2 \pi$ is a rational number, $\omega_{0}=2 \pi q / p$. The difference equation (6] becomes

$$
z_{n+1}=e^{-\mathrm{i} \omega_{0}} z_{n}+\varepsilon\left[\frac{\mathrm{i} \exp \left(-\mathrm{i} \omega_{0} / 2\right)}{2 \sin \omega_{0}} g\left(u_{n}\right)-\mathrm{i} \delta z_{n}\right]+\mathrm{O}\left(\varepsilon^{2}\right) .
$$

With the same procedure as we have followed in the previous subsections, we can derive the renormalization group equation in the case of $n \gg 1$ as follows:

$$
\begin{aligned}
& \frac{\mathrm{d} z_{n}}{\mathrm{~d} \tau}=\mathrm{i} \frac{1}{2 \sin \omega_{0}}\left[\sum_{l \geq 1, m \geq 0} A_{2 l+m p-1} 2 l+m p-1\right. C_{l+m p}\left|z_{n}\right|^{2(l-1)} z_{n}^{m p+1} \\
&+\sum_{l \geq 0, m \geq 1} A_{2 l+m p-1} 2 l+m p-1 \\
&
\end{aligned}
$$

For simplicity, let us consider such a case as follows:

$$
\begin{aligned}
g\left(u_{n}\right) & :=b u_{n}^{p-1}, \\
& =b \sum_{l=0}^{p-1}{ }_{p-1} C_{l} \exp [-\mathrm{i}(2 l-p+1) \omega / 2] z_{n}^{l}{\overline{z_{n}}}^{p-l-1},
\end{aligned}
$$

where $b$ is a parameter. Then the renormalization group equation (30), which describes a long-time behaviour, becomes

$$
\frac{\mathrm{d} z_{n}(\tau)}{\mathrm{d} \tau}=\alpha \frac{\mathrm{i} b}{2 \sin \omega_{0}}{ }_{p-1} C_{\frac{p}{2}} z_{n}^{\frac{p}{2}} \bar{z}_{n}^{\frac{p}{2}-1}+\frac{\mathrm{i} b}{2 \sin \omega_{0}}{\overline{z_{n}}}^{p-1}-\mathrm{i} \delta z_{n},
$$

where $\alpha=1$ when $p$ is an even and $\alpha=0$ when $p$ is an odd. This is a Hamiltonian system whose Hamiltonian is

$$
H=\alpha \frac{\mathrm{i} b}{p \sin \omega_{0}}{ }_{p-1} C_{\frac{p}{2}}\left|z_{n}\right|^{p}+\frac{\mathrm{i} b}{2 p \sin \omega_{0}}\left(z_{n}^{p}+{\overline{z_{n}}}^{p}\right)-\mathrm{i} \delta\left|z_{n}\right|^{2} .
$$

At fixed points $z_{n}$ satisfies

$$
\alpha \frac{\mathrm{i} b}{2 \sin \omega_{0}}{ }_{p-1} C_{\frac{p}{2}} z_{n}^{\frac{p}{2}}{\overline{z_{n}}}^{\frac{p}{2}-1}+\frac{\mathrm{i} b}{2 \sin \omega_{0}}{\overline{z_{n}}}^{p-1}-\mathrm{i} \delta z_{n}=0 .
$$

Setting $z_{n}:=r e^{\mathrm{i} \theta}$ where $r$ and $\theta \in \mathbb{R}$,

$$
\sin (p \theta)=0, \text { and } r^{p-2}=\frac{2 \delta \sin \omega_{0}}{b\left(\alpha_{p-1} C_{\frac{p}{2}}+\cos (p \theta)\right)}>0,
$$

which reads

$$
\begin{array}{ll}
r^{p-2}=\frac{2 \delta \sin \omega_{0}}{b\left(\alpha_{p-1} C_{\frac{p}{2}}+1\right)} & \text { for } \theta=\frac{\pi}{p} 2 l, \\
r^{p-2}=\frac{2 \delta \sin \omega_{0}}{b\left(\alpha_{p-1} C_{\frac{p}{2}}-1\right)} & \text { for } \theta=\frac{\pi}{p}(2 l+1),
\end{array}
$$

where $l=0,1, \ldots, p-1$. When $p$ is even number which satisfies $p \geq 4$ and $b \delta>0$, there are $2 p$ fixed points. In this case, it can be shown that the half of them are elliptic, while the others are hyperbolic, and the Poincaré-Birkoff bifurcation occurs. Here the resonance structure in this case consists of a chain of $p$ resonant islands. 


\section{Concluding remarks}

The renormalization group method with Lie symmetry is developed and applied to difference equations such as symplectic map. When we apply the conventional renormalization group method to a singular perturbation problem of difference equations, it is difficult to find how to construct the renormalization group equation, which is a continuous differential equation. In this paper, we succeed in the consistent derivation of a renormalization group equation to a difference equation by employing the theory of Lie symmetry. Our results is significant in the sense that a long-time behavior of a perturbed difference equation is described by a continuous differential equation, that is the renormalization group equation. Furthermore, for the case of a simplistic map, it is shown the renormalization group equation becomes a Hamiltonian system. Then the phase space structure can be described by the Hamiltonian. As an application of our theory, we analyze the Poincaré-Birkoff bifurcation in a 2-D symplectic map and the condition of the Poincaré-Birkoff bifurcation is given to the 2-D symplectic map.

\section{References}

[1] Chen L Y, Goldenfeld N and Oono Y 1996 Phys. Rev. E54 376

[2] Goto S, Masutomi Y and Nozaki K 1999 Progress of Theoretical Physics 102471

[3] Iwasa M and Nozaki K 2006 Progress of Theoretical Physics 116605

[4] Maruo T, Goto S and Nozaki K 2004 Progress of Theoretical Physics 111463

[5] Kano H and Nozaki K 2007 Chaos, Solitons and Fractals 32215

[6] Olver P J 1986 Applications of Lie Groups to Differential Equations (Springer-Verlag New York) 